\title{
El Programa DOCENTIA: Fundamentos, Desarrollo e Implicaciones
}

\author{
The DOCENTIA Programme: \\ Basis, Development and Implications
}

DOI http://dx.doi.org/10.1344/REYD2018.17.23483

\section{Eduardo García-Jiménez}

Catedrático de Universidad de Métodos de Investigación y Diagnostico en Educación Miembro de Comisión de Evaluación del Programa Docentia.

Facultad de Ciencias de la Educación.

Universidad de Sevilla.

Calle Pirotecnia, s/n, 41013. Sevilla

E-mail:egarji@us.es

\section{María -José Rodríguez -Conde}

Catedrática de Universidad de Métodos de Investigación y Diagnóstico en Educación.

Directora del Instituto Universitario de Ciencias de la Educación (IUCE)

Universidad de Salamanca.

Paseo Canalejas, 169. 37008-Salamanca

E-mail: mjrconde@usal.es 
Resumen: Este artículo responde al objetivo de mostrar el desarrollo histórico de un programa de apoyo para la evaluación de la actividad docente del profesorado universitario denominado DOCENTIA, desde su origen, sus objetivos y su desarrollo en las instituciones de educación superior españolas. En primer lugar, comenzamos con la exposición de los antecedentes sobre evaluación docente en las instituciones de educación superior, ligadas al desarrollo de la Ley de Reforma Universitaria, hasta la aprobación de la vigente Ley de Universidades (LOMLOU, 2007). A continuación, describimos la naturaleza y sentido de la herramienta DOCENTIA en el nuevo contexto universitario, adaptado a las exigencias de garantía de calidad de las instituciones. Posteriormente, presentamos un análisis comparado de los diecisiete modelos de evaluación de la actividad docente certificados hasta diciembre de 2017 por las agencias de evaluación, a partir de criterios objetivos. A continuación, exponemos el desarrollo que ha seguido el programa en términos cuantitativos y cualitativos en la Universidad española. Finalizamos, planteando una reflexión sobre las implicaciones que en la práctica ha producido la aplicación de la herramienta de evaluación docente DOCENTIA, en la mejora de la función docente de la actividad universitaria.

Palabras claves: Evaluación de docencia, profesorado universitario, Universidad, Programa DOCENTIA, Garantía de calidad

Abstract: This paper aims to show the historical development of a programme supporting the assessment of the teaching activities of university professors called DOCENTIA, covering its origins, aims, and implementation within Spanish higher education institutions. Firstly, we will explain the antecedents on teacher assessment in higher education institutions, which are linked to the development of the University Reform Act, and up to the current Act on Universities (LOMLOU, 2007). Next, we will describe the nature of DOCENTIA as a tool adapted to the quality assurance demands in the new university context. Following this, we will present an analysis comparing the seventeen models of teacher assessment which were certified as of December 2017 by the assessment agencies based on objective

criteria. Then, we will explain the development of the programme in both qualitative and quantitative terms within the Spanish university system. We will finish off by reflecting on the practical implications of the teaches assessment tool DOCENTIA in the improvement of the teaching practice within Spanish universities.

Keywords: Teaching evaluation, University, DOCENTIA program, university teaching staff, quality assurance. 
El Programa DOCENTIA:

Fundamentos, Desarrollo e Implicaciones

The DOCENTIA Programme:

Basis, Development and Implications

1. Antecedentes del programa DOCENTIA: Encuestas de evaluación docente por los estudiantes y evaluación institucional

El recorrido histórico de la evolución de la evaluación de la docencia universitaria en nuestro entorno va unido al desarrollo democrático de la institución universitaria; comenzando por la aprobación de la Constitución (1978), hasta la normativa derivada de la actual Ley de Universidades ${ }^{1}$. Para entender el momento actual del proceso de evaluación de la docencia en las Universidades, es conveniente considerar su evolución histórica. Este recorrido va a ir ligado al proceso democratizador de la institución universitaria, en un primer momento; $y$, posteriormente, a su incorporación a la acordada estructura universitaria europea (Espacio Europeo de Educación Superior). Si la Constitución proporciona el marco de autonomía necesario para el impulso de la estructura universitaria moderna, también va a llevar asociado la rendición de cuentas a la sociedad. Y si con el acuerdo de Ministros de Educación europeos sobre Educación Superior entramos en Europa, asumimos también la cultura de la garantía de calidad para nuestros estudiantes y para la sociedad, en general (Rodríguez Conde, 2011).

\footnotetext{
1 LOMLOU, 2007: LEY ORGÁNICA 4/2007, de 12 de abril, por la que se modifica la Ley Orgánica 6/2001, de 21 de diciembre, de Universidades, BOE de 13 de abril de 2007. 
En esta breve historia de la evaluación de la docencia universitaria, algunos de los investigadores pioneros en España, Tejedor y Jornet (2008), nos recordaron, hace unos años, los cinco momentos clave que han ido configurando la situación actual de este proceso:

a) Evaluaciones de satisfacción de estudiantes hacia la docencia por profesor y asignatura, solicitadas por las universidades a agentes externos en los años 80 (Tejedor Tejedor, 1990), a partir del artículo 45.3 de la Ley de Reforma Universitaria (1983), donde se deja autonomía a la Universidad para organizar el proceso de evaluación docente del profesorado.

b) Implantación del R.D. 1086/1989, de 28 de agosto, sobre retribuciones del profesorado universitario (BOE de 9 de septiembre de 1989).

c) Desarrollo del Plan Nacional de Evaluación de la Calidad de las Universidades (1995²), desde la Secretaria de Estado de Universidades (Miguel Díaz, 1999; Vidal, 1999).

d) Puesta en marcha del sistema de acreditación de profesores contratados y la habilitación nacional de funcionarios a partir de la Ley Orgánica de Universidades (LOU ${ }^{3}$, 2001) y posteriormente, acreditación del profesorado contratado y funcionario (LOMLOU, 2007) .

e) Creación de la Agencia Nacional de Evaluación de la Calidad y Acreditación, en 2001 por la $\mathrm{LOE}^{4}$ y de las once agencias autonómicas de evaluación universitaria.

A estos cinco momentos habría que añadir un sexto hito, actualmente, con el diseño e implantación, por parte de la mayoría de las Universidades, del Programa DOCENTIA (ANECA, 2015), dentro del sistema de garantía de calidad de las enseñanzas universitarias.

\footnotetext{
2 Real Decreto 1947/1995, de 1 de diciembre, por el que se establece el Plan Nacional de Evaluación de la Calidad de las Universidades (BOE de 9 de diciembre de 1995).

3 Ley Orgánica 6/2001, de 21 de diciembre, de Universidades (BOE de 24 de diciembre de 2001).

4 Artículo 32 de la LOU. 
Pues bien, en los primeros años de los 90, surgen algunos trabajos de investigación, sobre todo en formato de Tesis Doctorales, en torno a los estudios evaluativos realizados sobre la satisfacción del estudiante hacia la actividad docente en cada una de las asignaturas (González Such, 1998; Rodríguez Conde, 1994). Constituyeron las primeras experiencias de recogida de información válida y fiable sobre algunas fuentes de información, en concreto, estudiantes y el propio profesor, para evaluar la docencia.

La preocupación de la investigación por obtener instrumentos de medida (cuestionarios cerrados en escalas tipo Likert) que respondieran a necesidades evaluativas y a la obtención de resultados en un tiempo lo más corto posible, se imponían frente a la necesaria consistencia métrica de sus medidas (González Such, 1998; Marsh, 1987; Peterson, 1984; Tejedor 1990). Este tema, la preocupación de los investigadores en educación por las garantías técnicas de los instrumentos de recogida de información de los estudiantes sobre la calidad docente percibida sigue siendo hoy en día motivo de análisis sistemático y reflexión psicométrica (LizasoainHernández, Etxeberria-Murgiondo, \& Lukas-Mujika, 2017; López Cámara, 2014; Pozo Muñoz, Giménez Torres, \& Nieto, 2009).

Por otro lado, en esta primera época, si se añadían otro tipo de fuentes e instrumentos de evaluación, como el autoinforme del profesor -en escasas ocasiones-, éste consistía en un cuestionario donde el propio docente expone sus condiciones de docencia y valora la adecuación de la selección de objetivos, contenidos, metodología y sistema de evaluación de estudiantes (Rodríguez Conde, 1994; Villa Sánchez, 1985).

Como nos exponía Murillo (2008), la normativa sobre retribuciones de 1989 (recordando dentro del desarrollo de la LRU, 1983, en relación a la evaluación del desempeño docente), introdujo un complemento específico por méritos docentes, para cada cinco años de dedicación a tiempo completo (quinquenio o tramo docente). Este complemento lo obtenían los docentes que alcanzaran una evaluación positiva efectuada por la universidad, a partir de unos criterios 
establecidos por el Consejo de Universidades (19905). Se regularon los criterios generales para la evaluación de la actividad docente del profesorado universitario y se fijaron tanto las actividades objeto de evaluación, como los instrumentos y fuentes de evaluación. Las actividades objeto de evaluación eran: el cumplimiento de los objetivos y obligaciones docentes; las actividades de formación y extensión universitaria; y la participación en actividades de la comunidad universitaria. Los instrumentos y fuentes de información quedaron definidos por: Informe del profesor sobre sus actividades; Encuesta de los alumnos; Informes de los órganos unipersonales o colegiados; y externos.

El Plan Nacional de Evaluación de la Calidad de las Universidades durante la década del 1995 al 2005, aproximadamente, de carácter voluntario para las instituciones, a pesar de sus limitaciones técnicas y de procedimiento, vino a impulsar una cultura de la evaluación en las Universidades y a preparar el siguiente proceso vinculado a la adaptación al EEES (Miguel Díaz, 1999; Rodriguez Espinar, 2013; Rodríguez Espinar, 2013)

La llegada del EEES y la Ley Orgánica que modifica la LOU, de 2007, ha impulsado que las universidades profundicen en una evaluación del desempeño docente, desde una perspectiva formativa, que ayude a la mejora de la actividad docente de las titulaciones, centros y de la Universidad en su conjunto (Stake, Contreras, \& Arbesú, 2011). El Programa de Apoyo a la Evaluación de la Actividad Docente del Profesorado (DOCENTIA), impulsado por la ANECA, es una prueba de ello.

\section{Naturaleza y fundamentos del programa DOCENTIA}

DOCENTIA puede definirse como un programa de "apoyo para la evaluación de la actividad docente del profesorado universitario" (ANECA, 2015, p. 5). Su nacimiento y posterior desarrollo han estado asociados a la promoción de la evaluación de la actividad docente entre las universidades. En consecuencia, DOCENTIA no es un modelo de evaluación al uso que defina qué es una actividad docente de calidad y

\footnotetext{
5 Resolución de 20 de junio de 1990. 
establezca los criterios y estándares que permitan medirla. De acuerdo con dicho programa, la definición del modelo de evaluación corresponde a cada universidad.

Cada una de las universidades participantes en el programa DOCENTIA establece la finalidad y consecuencias de la evaluación, concreta las dimensiones objeto de evaluación, precisa los criterios y estándares de evaluación, determina los instrumentos a utilizar para recoger información sobre la actividad docente del profesorado y elabora un protocolo de valoración de dicha información. De igual modo, cada universidad define un proceso de evaluación en el que se especifican las actividades a realizar, de acuerdo con un calendario que fija la periodicidad y duración de la evaluación. En dicho proceso, también se precisan los órganos encargados de aplicar el protocolo de evaluación, de atender las reclamaciones planteadas por el profesorado evaluado y de aplicar el resultado de la evaluación.

El papel de las agencias de evaluación participantes en el programa DOCENTIA es orientar y apoyar a las universidades en el diseño, aplicación y mejora de sus modelos de evaluación de la actividad docente del profesado, a través del "desarrollo de la colaboración técnica de las agencias de calidad universitaria con las universidades y las administraciones educativas de las Comunidades Autónomas" (ANECA, 2015, p. 4). Para ello, el programa DOCENTIA ofrece a la universidad la posibilidad de una evaluación externa en la que una agencia valora el diseño de su modelo de evaluación y, en su caso, lo certifica. La universidad también puede someter a seguimiento la aplicación de su modelo, por parte de la agencia correspondiente. De igual modo, la universidad puede solicitar la acreditación de su modelo de evaluación, cuando la ejecución de este satisface los criterios fijados en el programa DOCENTIA. Conforme a la definición del propio programa, uno de los valores añadidos que podría proporcionar DOCENTIA a las universidades es la retroalimentación sobre los procesos de evaluación para la certificación, del seguimiento y de la acreditación de los modelos.

El programa DOCENTIA se asienta sobre varios principios, a partir de los que se orienta el trabajo de las universidades y se articula el proceso de evaluación que deben llevar a cabo las agencias. Entre dichos principios cabe destacar cuatro. El 
primer principio vendría a señalar que la evaluación de la actividad docente tiene que ser comprensiva, de modo que en ella están contempladas las actividades -"a clase vacía" y "a clase llena" - que realiza el profesorado para facilitar el aprendizaje de los estudiantes, así como los resultados de dichas actividades. Para dar cumplimiento a este principio, DOCENTIA establece que los modelos de evaluación desarrollados por las universidades deben recoger, al menos, tres dimensiones: la planificación de la docencia, el desarrollo de la enseñanza y los resultados. En la dimensión planificación de la docencia se evalúan aquellas actividades desarrolladas por el profesorado referidas a la organización y la coordinación docentes, así como las estrictamente vinculadas al diseño de las actividades de enseñanza y aprendizaje (resultados previstos, actividades, recursos y procedimientos de evaluación) en las asignaturas de uno o varios planes de estudios. En la dimensión desarrollo de la enseñanza se evalúan las actividades de enseñanza, una vez desarrolladas, así como los procedimientos de evaluación del aprendizaje que han sido utilizados. Y, en la dimensión resultados se evalúa el nivel de logro de los objetivos formativos por parte de los estudiantes y las actividades de revisión y mejora llevadas a cabo por el profesorado.

En los documentos que concretan el programa DOCENTIA, queda explicitado que la dedicación del profesorado (en número de créditos o en el nivel de enseñanza impartida) constituye un requisito y no una dimensión que deba ser objeto de evaluación.

Los modelos han de estar centrados en la evaluación de la calidad de la actividad, no en el cumplimiento de las obligaciones docentes, que son la condición necesaria para poder evaluar la calidad del desempeño, ni en el mero sumatorio de méritos docentes. (ANECA, 2015, p. 14)

El programa deja a criterio de las universidades la posibilidad de complementar dichas dimensiones con otras relacionadas con la docencia, como por ejemplo la innovación o la investigación sobre la docencia.

Un segundo principio alude a la participación de la comunidad universitaria en el proceso de evaluación. De acuerdo con el programa DOCENTIA, el diseño del modelo 
de evaluación, el proceso establecido para aplicarlo, su propia ejecución y sus consecuencias deben estar inextricablemente unidos a los procesos, los órganos de gobierno y las personas que integran una universidad. Así, el modelo de evaluación definido por una universidad debe ser aprobado conforme a lo recogido en sus Estatutos y coherente con su plan estratégico, en lo relativo a la política de personal docente (evaluación, promoción, incentivos, formación, etc.). De igual modo, el proceso de evaluación debe ser público de modo que toda la comunidad universitaria debe conocer qué es, cómo se lleva a cabo y cuáles son las consecuencias de la evaluación; y, en los órganos de evaluación deben estar representados responsables académicos, profesores y estudiantes. Los resultados del proceso de evaluación (especialmente el Informe institucional) también deben ser difundidos y el propio proceso sometido a valoración por parte de profesores y estudiantes; los resultados de las evaluaciones individuales (los informes individuales), al menos deben ser del conocimiento de los responsables académicos encargados de tomar las decisiones que se deriven del proceso de evaluación.

El tercer principio está referido a la validez y fiabilidad de las evaluaciones. Este principio vendría a establecer que las evaluaciones deben ser útiles; es decir, deben tener consecuencias acordes con las finalidades del modelo elaborado por cada universidad (mejora, reconocimiento, promoción, etc.) y deben estar libres de sesgo. En este último caso, se indica que "la evaluación de la actividad docente del profesorado debe estar basada necesariamente en al menos tres fuentes de información" (ANECA, 2015, p. 17); es decir, en las valoraciones de profesores, estudiantes y responsables académicos (aunque la universidad podría incorporar las valoraciones de egresados y empleadores). La información a partir de dichas fuentes se obtiene mediante encuestas, autoinformes, informes, documentación y otros procedimientos (p.e. grupos de discusión, observaciones realizadas por pares) que valoran cada una de las dimensiones de la actividad docente del profesorado. De este modo, se garantiza una triangulación de la información recogida sobre una misma dimensión de evaluación.

Un cuarto principio que subyace al programa DOCENTIA es el de la sostenibilidad de los modelos de evaluación. Conforme a lo establecido en los diferentes documentos 
que conforman dicho programa, los modelos elaborados por las universidades deben ser viables y garantizar una continuidad de las actuaciones emprendidas. La viabilidad de los modelos propuestos por las universidades debe concretarse en su capacidad para ser aplicados a la población objeto de estudio, en el tiempo que necesitan para completar un ciclo anual o plurianual de evaluación y en los recursos que precisan para ejecutarse. En consecuencia, "deben garantizar la agilidad y eficiencia del proceso" (ANECA, 2015, p. 19) de modo que la evaluación no resulte excesivamente compleja y burocrática. La sostenibilidad hace referencia a la posibilidad de que las evaluaciones llevadas a cabo conforme a dichos modelos se mantengan en el tiempo y no se vean sometidas a rediseños o cambios que afecten a sus elementos esenciales, de modo que sirvan de referencia a la evaluación y formación a lo largo de la carrera docente del profesorado.

\section{Los modelos de evaluación de la actividad docente propuestos por las universidades}

El análisis de los modelos del programa DOCENTIA se ha centrado en aquellos modelos cuya implantación ha sido certificada por ANECA o por las agencias de evaluación autonómicas. La no incorporación a dicho análisis de los modelos cuyo diseño está certificado, pero no implantado, está motivada por el hecho de que dichos modelos están aún en fase experimental y pueden sufrir importantes modificaciones.

La Tabla 1 presenta una comparación de los 17 modelos de evaluación de la actividad docente certificados hasta diciembre de 2017 por las agencias de evaluación, según lo publicado en la web de ANECA con relación al programa DOCENTIA. La titularidad de las universidades que han certificado su modelo de evaluación es mayoritariamente pública $(70,59 \%)$. Esta preponderancia también se mantiene en términos relativos, ya que 12 de las de 33 universidades públicas (36,37\%) que participan en el programa DOCENTIA han certificado su modelo mientras que sólo lo han hecho 5 universidades privadas de las 21 (23,81\%) que participan en dicho programa. La tipología de las universidades españolas, en función del tamaño de su plantilla, está representada entre las universidades que han certificado un modelo 
de evaluación de modo que se encuentran universidades públicas con más de 5,000 profesores, públicas entre 1,000 y 3,000 profesores y con menos de 1,000 profesores, privadas con un millar de profesores y con sólo unos cientos.

La participación del profesorado en la evaluación de su actividad docente es mayoritariamente voluntaria $(64,71 \%)$, especialmente entre las universidades públicas, y es obligatoria sobre todo en las universidades privadas. El alcance de la evaluación sigue un patrón mayoritario: el profesorado objeto de evaluación es sobre todo el que mantiene una vinculación permanente con la universidad, si bien también de forma progresiva se ha incorporado la práctica totalidad del profesorado. Los profesores, en el patrón más común, evalúan su actividad docente cada 5 años a partir de la información que se recoge anualmente. Esta periodicidad suele verse voluntariamente reducida cuando el profesorado participa en procesos de promoción.

El modelo de evaluación certificado más habitual es aquel que se ajusta exclusivamente a las dimensiones establecidas en el programa DOCENTIA (planificación, desarrollo y resultados), y al tipo de evidencias (cuestionarios, autoinformes, informes de responsables académicos y documentos) y fuentes sugeridas por dicho programa (estudiantes, profesores y responsables académicos). Esta es la razón por la que en la Tabla 1 únicamente hemos recogido los elementos diferenciadores (las celdas vacías indican que se satisfacen los requerimientos del programa); incluso, alguno de los elementos recogido en dicha tabla, como la utilización del rendimiento académico de los alumnos para valorar la actividad docente del profesorado resulta mayoritario cuando se consideran los modelos certificados y no certificados. El desarrollo profesional del profesorado -entendido como la suma de innovación docente y formación- es la dimensión que las universidades han incorporado con más frecuencia al programa. Los procedimientos de recogida de información -con la excepción del uso de entrevistas, rúbricas y algún tipo de informe adicional- siguen la tipología establecida en el programa DOCENTIA al igual que las evidencias, con la excepción del ya comentado rendimiento académico. 
Los datos sobre los que se apoya la valoración de la actividad docente, a tenor de lo comentado en el párrafo anterior, suelen ser de diferente naturaleza: cuantitativos, en forma de indicadores u obtenidos a partir de los cuestionarios de satisfacción completados por los estudiantes; y, cualitativos, de tipo categórico o textual procedentes de los autoinformes del profesorado y de los informes de responsables académicos. Algunos modelos, como los de la Universitat Politécnica de València o la Jaume I se apoyan sobre todo en el uso de indicadores.

La mejora de la actividad docente del profesorado, especialmente en los docentes con valoraciones más bajas, es la consecuencia más característica que puede observarse entre los modelos certificados. Esta consecuencia está presente en todos los modelos por lo que no se ha incorporado a la Tabla 1 (ver celdas vacías). La consecuencia más habitual es la vinculación entre los resultados deficientes en la evaluación y el establecimiento de actuaciones genéricas de formación, en las que el profesorado participa de forma voluntaria. En esta tabla se han destacado aquellas consecuencias que diferencian a un modelo en particular frente a la mayoría de los modelos certificados. Así, con relación a la formación del profesorado, la Universidad Europea Miguel de Cervantes la considera obligatoria para los profesores con una evaluación negativa; la Universitat de Lleida prevé un plan integral de formación de dicho profesorado, la Universitat Pompeu Fabra establece planes de formación individualizados y negociados y la Universidad Antonio de Nebrija realiza un seguimiento semestral del plan de mejora del profesorado.

Los quinquenios docentes son una consecuencia compartida por muchos modelos, especialmente en el caso de las universidades públicas catalanas. En términos de remuneración económica, algunas universidades como la Universidad Francisco de Vitoria o Internacional de Catalunya ofrecen complementos retributivos a su profesorado mejor evaluado mientras que otras consideran los resultados de la evaluación de la actividad docente como indicador de la financiación de los centros (Autónoma de Barcelona y Barcelona) o de los centros y departamentos (Burgos y Jaume I). En este ámbito, también cabría situar a aquellos modelos que incorporan otro tipo de incentivos como el acceso gratuito a actividades deportivas y formación. 
Los resultados de la evaluación constituyen un mérito en los procesos de promoción del profesorado (especialmente en las universidades públicas) o de renovación de contratos, especialmente como concursante, pero también como miembro de la comisión evaluadora. De igual modo, dichos resultados se toman en consideración en las convocatorias de proyectos de innovación docente promovidas por algunas universidades, como la Autónoma de Barcelona o la Universitat de Barcelona. La concesión de licencias sabáticas o de solicitudes de movilidad también se asocia a los resultados de la evaluación en algunas universidades, como la Universidad Miguel de Cervantes.

Tabla 1. Comparación entre los modelos de evaluación DOCENTIA implantados y certificados.

\begin{tabular}{|c|c|c|c|c|c|c|}
\hline Universidad & Titularidad & $\begin{array}{l}\text { Número }^{1} \\
\text { profesores }\end{array}$ & $\begin{array}{c}\text { Participación } \\
\text { profesorado }\end{array}$ & $\begin{array}{c}\text { Evidencias/ } \\
\text { dimensiones } \\
\text { diferenciadora }^{2}\end{array}$ & $\begin{array}{c}\text { Valoración a } \\
\text { partir de... }\end{array}$ & $\begin{array}{l}\text { Consecuencias } \\
\text { diferenciadora }^{3}\end{array}$ \\
\hline $\begin{array}{l}\text { Antonio de } \\
\text { Nebrija }\end{array}$ & Privada & 348 & Obligatoria & - & $\begin{array}{c}\text { Datos } \\
\text { ordinales y } \\
\text { cualitativos }\end{array}$ & $\begin{array}{l}\text { Seguimiento } \\
\text { semestral plan de } \\
\text { mejora profesor }\end{array}$ \\
\hline $\begin{array}{l}\text { Autónoma } \\
\text { de } \\
\text { Barcelona }\end{array}$ & Pública & 3,455 & Voluntaria & $\begin{array}{l}\text { Dimensión: } \\
\text { Desarrollo } \\
\text { profesional, } \\
\text { Tasas de } \\
\text { rendimiento } \\
\text { asignaturas }\end{array}$ & $\begin{array}{c}\text { Rúbrica, } \\
\text { datos } \\
\text { ordinales y } \\
\text { datos } \\
\text { cualitativos }\end{array}$ & $\begin{array}{l}\text { Quinquenios, } \\
\text { mérito solicitud } \\
\text { innovación, } \\
\text { miembro comisión } \\
\text { concurso plazas, } \\
\text { licencias sabáticas } \\
\text { y emérito, } \\
\text { excelencia. } \\
\text { Financiación del } \\
\text { centro }\end{array}$ \\
\hline $\begin{array}{l}\text { Autónoma } \\
\text { de Madrid }\end{array}$ & Pública & 2,498 & Voluntaria & - & $\begin{array}{l}\text { Indicadores } \\
\text { y datos } \\
\text { cualitativos }\end{array}$ & $\begin{array}{l}\text { Informe de } \\
\text { retroalimentación } \\
\text { personalizado }\end{array}$ \\
\hline Barcelona & Pública & 5,182 & Voluntaria & $\begin{array}{l}\text { Resultados } \\
\text { académicos }\end{array}$ & $\begin{array}{c}\text { Datos } \\
\text { cualitativos, } \\
\text { indicadores }\end{array}$ & $\begin{array}{l}\text { Quinquenios, } \\
\text { mérito solicitud } \\
\text { innovación, } \\
\text { promoción, } \\
\text { miembro comisión } \\
\text { concurso plazas, } \\
\text { emérito, } \\
\text { excelencia. } \\
\text { Financiación del } \\
\text { centro }\end{array}$ \\
\hline Burgos & Pública & 762 & Voluntaria & - & \begin{tabular}{|} 
Datos \\
cualitativos \\
$\mathrm{y}$ \\
cuantitativos
\end{tabular} & $\begin{array}{c}\text { Baremos de plazas, } \\
\text { financiación } \\
\text { centros y } \\
\text { departamentos }\end{array}$ \\
\hline
\end{tabular}


El Programa DOCENTIA: Fundamentos, Desarrollo e Implicaciones.

\begin{tabular}{|c|c|c|c|c|c|c|}
\hline Universidad & Titularidad & $\begin{array}{l}\text { Número }^{1} \\
\text { profesores }\end{array}$ & $\begin{array}{c}\text { Participación } \\
\text { profesorado }\end{array}$ & $\begin{array}{c}\text { Evidencias/ } \\
\text { dimensiones } \\
\text { diferenciadora }^{2}\end{array}$ & $\begin{array}{c}\text { Valoración a } \\
\text { partir de... }\end{array}$ & $\begin{array}{l}\text { Consecuencias } \\
\text { diferenciadora }\end{array}$ \\
\hline $\begin{array}{l}\text { Europea } \\
\text { Miguel de } \\
\text { Cervantes }\end{array}$ & Privada & 164 & Obligatoria & $\begin{array}{c}\text { Informe Unidad } \\
\text { de Innovación } \\
\text { Docente y } \\
\text { Tutorial }\end{array}$ & \begin{tabular}{|c} 
Datos \\
cualitativos \\
$\mathrm{y}$ \\
cuantitativos
\end{tabular} & $\begin{array}{c}\text { Acceso gratuito a } \\
\text { actividades depor- } \\
\text { tivas y formación. } \\
\text { Evaluación } \\
\text { negativa conlleva } \\
\text { formación } \\
\text { obligatoria }\end{array}$ \\
\hline $\begin{array}{l}\text { Francisco de } \\
\text { Vitoria }\end{array}$ & Privada & 525 & Voluntaria & - & \begin{tabular}{|} 
Rúbrica, \\
datos \\
cualitativos \\
y \\
cuantitativos
\end{tabular} & $\begin{array}{c}\text { Complementos } \\
\text { económicos } \\
\text { anuales }\end{array}$ \\
\hline Girona & Pública & 1,204 & Voluntaria & $\begin{array}{l}\text { Tasas de } \\
\text { rendimiento } \\
\text { asignaturas }\end{array}$ & $\begin{array}{c}\text { Datos } \\
\text { ordinales, } \\
\text { indicadores } \\
\text { y datos } \\
\text { cualitativos }\end{array}$ & Quinquenios \\
\hline Jaume I & Pública & 1,221 & Obligatoria & $\begin{array}{l}\text { Tasas de } \\
\text { rendimiento } \\
\text { asignaturas }\end{array}$ & Indicadores & $\begin{array}{l}\text { Promociones y } \\
\text { renovaciones de } \\
\text { contratos, } \\
\text { presupuestos } \\
\text { dptos. y centros, } \\
\text { incentivos } \\
\text { económicos }\end{array}$ \\
\hline $\begin{array}{l}\text { Internaciona } \\
\text { l de } \\
\text { Catalunya }\end{array}$ & Privada & 512 & Obligatoria & $\begin{array}{c}\text { Dimensión: } \\
\text { Investigación en } \\
\text { docencia }\end{array}$ & $\begin{array}{c}\text { Datos } \\
\text { cualitativos } \\
y \\
\text { cuantitativos }\end{array}$ & $\begin{array}{l}\text { Renovación } \\
\text { contratos, } \\
\text { incentivos } \\
\text { económicos }\end{array}$ \\
\hline Lleida & Pública & 1,041 & Voluntaria & $\begin{array}{l}\text { Tasas de } \\
\text { rendimiento } \\
\text { asignaturas }\end{array}$ & $\begin{array}{c}\text { Datos } \\
\text { ordinales y } \\
\text { cualitativos }\end{array}$ & $\begin{array}{c}\text { Quinquenios, Plan } \\
\text { integral de } \\
\text { formación del } \\
\text { profesorado }\end{array}$ \\
\hline $\begin{array}{l}\text { Politécnica } \\
\text { de Catalunya }\end{array}$ & Pública & 2,541 & Voluntaria & $\begin{array}{l}\text { Dimensión: } \\
\text { Desarrollo } \\
\text { profesional, }\end{array}$ & $\begin{array}{c}\text { Indicadores } \\
\text { y datos } \\
\text { cualitativos }\end{array}$ & $\begin{array}{c}\text { Quinquenios, } \\
\text { contratación, } \\
\text { estabilización, } \\
\text { promoción, } \\
\text { miembro comisión } \\
\text { concurso plazas, } \\
\text { licencias sabáticas } \\
\text { y permisos de } \\
\text { movilidad }\end{array}$ \\
\hline $\begin{array}{l}\text { Politécnica } \\
\text { de València }\end{array}$ & Pública & 2.589 & Obligatoria & $\begin{array}{l}\text { Tasa de } \\
\text { rendimiento } \\
\text { asignaturas }\end{array}$ & Indicadores & Quinquenios \\
\hline $\begin{array}{l}\text { Pompeu } \\
\text { Fabra }\end{array}$ & Pública & 877 & Voluntaria & $\begin{array}{l}\text { Tasas de } \\
\text { rendimiento } \\
\text { asignaturas }\end{array}$ & \begin{tabular}{|} 
Datos \\
ordinales, \\
indicadores \\
y datos \\
cualitativos
\end{tabular} & $\begin{array}{c}\text { Entrevista y planes } \\
\text { de formación } \\
\text { individualizados y } \\
\text { negociado con el } \\
\text { profesor/a. }\end{array}$ \\
\hline
\end{tabular}


El Programa DOCENTIA: Fundamentos, Desarrollo e Implicaciones.

\begin{tabular}{|l|c|c|c|c|c|c|}
\hline Universidad & Titularidad & $\begin{array}{c}\text { Número }{ }^{1} \\
\text { profesores }\end{array}$ & $\begin{array}{c}\text { Participación } \\
\text { profesorado }\end{array}$ & $\begin{array}{c}\text { Evidencias/ } \\
\text { dimensiones } \\
\text { diferenciadora }\end{array}$ & $\begin{array}{c}\text { Valoración a } \\
\text { partir de... }\end{array}$ & $\begin{array}{c}\text { Consecuencias } \\
\text { diferenciadora }\end{array}$ \\
\hline Ramón Llull & Privada & 1,008 & Obligatoria & $\begin{array}{c}\text { Dimensión: } \\
\text { Actualización e } \\
\text { Innovación } \\
\text { Docente; } \\
\text { Entrevistas }\end{array}$ & $\begin{array}{c}\text { Datos } \\
\text { ordinales y } \\
\text { datos } \\
\text { cualitativos }\end{array}$ & Promoción \\
\hline $\begin{array}{l}\text { Rey Juan } \\
\text { Carlos }\end{array}$ & Pública & 1,351 & Voluntaria & $\begin{array}{c}\text { Informe de } \\
\text { Centro }\end{array}$ & $\begin{array}{c}\text { Indicadores } \\
\text { y datos } \\
\text { cualitativos }\end{array}$ & \\
\hline $\begin{array}{l}\text { Rovira i } \\
\text { Virgili }\end{array}$ & Pública & 1,673 & Voluntaria & $\begin{array}{c}\text { Tasas de } \\
\text { rendimiento } \\
\text { cualitativos } \\
\text { y }\end{array}$ & Quinquenios \\
\hline
\end{tabular}

Fuente: Elaboración propia

${ }^{1}$ Datos del curso 2015-16 publicados por el Ministerio de Educación, Cultura y Deporte. Los datos incluyen al profesorado de los centros propios de la Universidad, de todas las categorías profesionales y de ambos sexos (recuperado de https://www.educacion.gob.es/educabase/menu.do?type=pcaxis\&path=/Universitaria/Personal/2015-

2016/PDI/3 Total Universidad\&file=pcaxis\&l=s0).

${ }^{2}$ En la tabla únicamente se recogen los elementos diferenciadoras ya que todas las universidades recogen evidencias de al menos tres colectivos (estudiantes, profesores y responsables), utilizando para ello tres métodos o instrumentos de recogida de información (cuestionario, autoinforme e informe de responsable).

${ }^{3}$ En la tabla únicamente figuran las consecuencias diferenciadoras dado que todas las universidades incorporar consecuencias orientadas a la mejora de la docencia y a la formación del profesorado.

\section{El desarrollo del programa DOCENTIA}

El programa DOCENTIA está vertebrado sobre dos ejes: las universidades y las agencias de evaluación. Las Universidades diseñan, implantan, revisan y mejoran sus modelos de evaluación mientras que las agencias revisan dichas actuaciones y, en su caso, reconocen la validez de las mismas conforme a los principios del propio programa.

En su papel de agentes externos, las agencias de evaluación realizan cuatro procesos básicos. Así, las agencias llevan a cabo una verificación del diseño del modelo de evaluación de la actividad docente elaborado por una universidad, cuando ésta lo solicita. Una vez verificado el modelo de evaluación de una universidad, las agencias realizan un seguimiento de su implantación. Finalmente, las agencias realizan una evaluación para la certificación del modelo cuando está completamente implantado y "cumple las especificaciones y directrices del programa DOCENTIA" (ANECA, 2015, p. 28). Dado que la certificación tiene una validez de 5 años, las agencias realizan evaluaciones periódicas que pueden conducir a la renovación de la certificación. 
La evaluación externa que realizan las agencias de los modelos de evaluación, en cualquiera de los procesos mencionados, se centra en el análisis de tres dimensiones básicas: estratégica, metodológica y de resultados. En la dimensión estratégica, la evaluación externa analiza los fundamentos y los objetivos del modelo de evaluación de la universidad. En la dimensión metodológica, el análisis considera las dimensiones, criterios y fuentes para la recogida de la información de dicho modelo. Por último, en la dimensión resultados, el foco de la evaluación está en los procedimientos para la toma de decisiones derivada de la evaluación, en la difusión de los resultados de la evaluación y en la revisión y mejora del proceso de evaluación de la actividad docente. En las dos últimas dimensiones, la evaluación que realizan las agencias se centra no sólo en el diseño propuesto por la universidad sino también en su implementación.

La evaluación externa toma en consideración un conjunto de requisitos básicos que deben satisfacer los modelos que son objeto de evaluación. Esos requisitos son diferentes en la verificación, el seguimiento, la certificación y la renovación de la certificación. Así, por ejemplo, la evaluación de la implantación de un modelo verificado no tiene lugar hasta que dicho modelo no se ha aplicado de forma experimental durante de al menos dos años, y la certificación de un modelo no es posible si una universidad no ha evaluado al 30\% del profesorado cuya actividad docente es objeto de objeto de evaluación.

Las agencias apoyan su valoración en una serie de criterios de evaluación que son comunes a la verificación, el seguimiento, la certificación y la renovación de los modelos propuestos por las universidades. Uno de dichos criterios considera la pertinencia del modelo de evaluación de la actividad docente para atender a los requerimientos, necesidades o prioridades establecidos en la legislación autonómica y en los estatutos, las planificaciones y las políticas de recursos humanos de la universidad que ha elaborado el modelo. De igual modo, otro criterio valora la adecuación del modelo de evaluación analizando las relaciones estructurales que mantienen sus principales componentes: dimensiones, criterios, fuentes de recogida de información y procedimientos. Un tercer criterio de evaluación es la coherencia de los elementos que concretan la aplicación del modelo 
(alcance, periodicidad, obligatoriedad, etc.) a sus objetivos y metas. Un cuarto criterio de evaluación, viabilidad y sostenibilidad analiza si el modelo propuesto cuenta con unos recursos y un modelo/una práctica de gestión permite llevar a cabo los procesos diseñados y alcanzar las finalidades previstas. Por último, se valora la eficacia de los modelos de evaluación de actividad docente considerando sus consecuencias (previstas o reales) y la difusión de sus resultados.

Las agencias desarrollan sus procesos de evaluación externa mediante una metodología de revisión por pares. Esta revisión se lleva a cabo por comisiones formadas por académicos, estudiantes y profesionales que elaboran informes de naturaleza técnica, a partir de los que las agencias toman decisiones sobre la calidad del diseño presentado por las universidades o su implementación. La selección, nombramiento y formación de los miembros de las comisiones se realiza por parte de las agencias conforme a los European Quality Standards and Guidelines (ESG, 2015). Estas comisiones tienen una estructura similar pero sus actuaciones difieren en función de los procesos de evaluación en los que participan; por ejemplo, únicamente en el proceso de certificación (y en su caso de renovación de la acreditación), las comisiones realizan una visita a las universidades para comprobar in situ la aplicación de un modelo.

Las actuaciones que deben llevar a cabo las comisiones de evaluación están procesualmente orientadas y se apoyan en diferentes protocolos que se recogen en un documento elaborado por ANECA y las agencias de evaluación de las comunidades autónomas (ANECA, 2015). En dicho documento se concretan los fundamentos, finalidad, dimensiones y criterios en los que se apoya la evaluación. También se incluye la secuenciación de las actuaciones de las comisiones en los procesos de verificación, seguimiento, certificación y renovación de la certificación, y sus correspondientes protocolos para la recogida de información, para la realización de la visita y la elaboración de los informes de evaluación.

Los resultados de las evaluaciones realizadas en el marco del programa DOCENTIA se recogen en la Tabla 2. Como puede verse en dicha tabla, de las 84 universidades existentes en el sistema universitario español, 71 participan en el programa 
DOCENTIA (85\%). Todas las universidades públicas, excepto la Universidad Internacional Menéndez Pelayo, participan en DOCENTIA ( $\mathrm{n}=50$ ) y representan el $70 \%$ del total de universidades en dicho programa. Las universidades privadas $(n=21)$ representan el 30\% de la participación en DOCENTIA y un 64\% sobre el total de universidades privadas $(n=33)$. En este sentido, hay un sesgo en la participación en DOCENTIA a favor de las universidades públicas.

Los resultados de las evaluaciones realizadas recogidos en la Tabla 2 indican que 64 de las 71 universidades participantes (90\%) tienen el diseño de su modelo de evaluación verificado; de las que no lo tienen, 3 son universidades públicas y 4 privadas. Entre los 64 modelos ya certificados, cabe distinguir los 25 que están en proceso de implantación con uno o más años de seguimiento que representa (o incluso un informe favorable previo a la certificación) y los 17 cuya implantación está certificada. Las 22 universidades restantes (34\%) acababan de verificar su modelo o no habían aportado datos sobre su implantación. Atendiendo a los datos cabe decir que el programa se ha completado en una de cada cinco universidades del sistema universitario (17/84), que ahora cuenta con un sistema certificado de evaluación de la actividad docente de su profesorado. Si sumamos los sistemas certificados y los que se encuentran en una fase avanzada de implantación, el impacto del programa se amplía a una de cada dos universidades (42/84).

Respecto a la titularidad de las universidades participantes, cabe destacar que las universidades públicas y privadas mantienen las mismas proporciones en los diferentes resultados obtenidos en la evaluación de sus modelos, por lo que no se aprecian sesgos en dichos resultados. Así, las universidades privadas representan un $30 \%$ del total, un $28 \%$ de los modelos verificados e implantados y un $29 \%$ de los certificados.

Tabla 2. Distribución de frecuencias correspondiente a los resultados del programa DOCENTIA.

\begin{tabular}{|l|l|c|c|c|c|}
\hline \multicolumn{2}{|c|}{ Universidades } & $\begin{array}{c}\text { Participan en el } \\
\text { programa }\end{array}$ & $\begin{array}{c}\text { Modelos } \\
\text { verificados }\end{array}$ & $\begin{array}{c}\text { Modelos en } \\
\text { implantación }\end{array}$ & $\begin{array}{c}\text { Modelos } \\
\text { Certificados }\end{array}$ \\
\hline Públicas & 51 & 50 & 46 & 18 & 12 \\
\hline Privadas & 33 & 21 & 18 & 7 & 5 \\
\hline
\end{tabular}




\begin{tabular}{|l|l|l|l|l|l|}
\hline Total & 84 & 71 & 64 & 25 & 17 \\
\hline
\end{tabular}

Fuente: Elaboración propia a partir de los resultados publicados en la página web de ANECA www.aneca.es/Programas-de-evaluacion/DOCENTIA/Resultados (Recuperado 13 de Enero de 2018).

Las agencias responsables de la evaluación de los diferentes modelos consideran que la participación de las universidades en el programa DOCENTIA presenta una escasa variación en los tres últimos años, de modo que en torno a un 15\% de ellas $(13 / 84)$ no termina de incorporarse al programa. De igual modo, "no se aprecia que, en términos generales, las universidades participantes estén evolucionando a través de las diferentes fases propuestas en DOCENTIA" (ANECA, 2017, p. 56).

\section{Las implicaciones del programa DOCENTIA: la evaluación de la docencia al servicio de la formación permanente y de la innovación en educación superior}

En este apartado final queremos revisar algunas consecuencias de la aplicación del programa DOCENTIA que deberían darse en la Universidad, según lo explicita el programa (ANECA, 2015), al marcar como requisito de certificación del propio programa, entre otros, "que las consecuencias que se derivan de su aplicación incidan en la mejora de la calidad de la docencia de la universidad".

Es cierto que en algunas Universidades que están implantando desde hace unos años el Programa DOCENTIA, por ejemplo, en la Universidad de Salamanca, los resultados del programa se han puesto a disposición de los responsables de distintos órganos académicos para apoyar la toma de decisiones en sus respectivos ámbitos (PROGRAMA DOCENTIA, 2016, p. 28). Los resultados del programa están contribuyendo en:

- Méritos en la promoción del profesor

- Diseño y acceso a actividades de formación

- Diseño y acceso a proyectos de innovación

- Asignación de responsabilidades docentes

- Ordenación de la planificación docente

- Diseño de planes de formación del profesorado.

- Diseño de planes de innovación docente 
En este sentido, Rodríguez Espinar (2013b) plantea que todo proceso de evaluación en la Universidad tiene sentido cuando se orienta en la dirección correcta: producir cambios encaminados al logro de una mayor calidad universitaria. Pues bien, veamos en qué aspectos concretos relativos a la docencia universitaria, podría observarse la influencia de la aplicación de un programa de evaluación docente.

Recordemos, en primer lugar, a nivel europeo, dentro de la estrategia de la Comisión Europea, que para apoyar la modernización de la educación superior en los Estados miembros, el 18 de junio de 2013, publica el primer informe ${ }^{6}$ del "Grupo de alto nivel para la modernización de la enseñanza superior de la Unión Europea” sobre la mejora de la calidad de la enseñanza y el aprendizaje en las universidades. Este grupo, ofrece dieciséis recomendaciones, de las que destacamos la segunda: "Every institution should develop and implement a strategy for the support and on-going improvement of the quality of teaching and learning, devoting the necessary level of human and financial resources to the task, and integrating this priority in its overall mission, giving teaching due parity with research." (European Commission, 2013, p. 27). En esta recomendación, donde se insiste en equiparar la prioridad de la investigación con la docencia en la Universidad, se menciona el esfuerzo del programa DOCENTIA en España (p. 28) por colaborar en el desarrollo de esta recomendación.

A nivel institucional, el apoyo a la docencia debe ser claramente establecido y, en consecuencia, la necesidad de relacionar Evaluación y Formación permanente del profesorado es ineludible. Algunas autoras, como Fernández Mach (2008), reflexionan sobre la necesidad de un marco o referente institucional en el diseño e implementación de planes de formación docente del profesorado universitario. Entre las condiciones del marco institucional, se encontrarían las políticas de incentivación y acreditación de la calidad de la docencia. Quiere decir que, para demostrar que la calidad de la docencia es un objetivo prioritario para una institución universitaria, es imprescindible que planifique y ponga en marcha

\footnotetext{
6 http://europa.eu/rapid/press-release_IP-13-554_es.htm 
acciones que motiven al profesorado para alcanzar el objetivo. Una iniciativa es el Programa DOCENTIA, con el fin de evaluar la calidad de la docencia y distinguir la excelencia de la misma.

Desde un punto de vista de la formación pedagógica del profesorado, a nivel individual, los retos a los que ha tenido que hacer frente en esta década de adaptación al EEES, le han impulsado- a una mayoría, no a todos o todas- a demandar formación permanente a sus instituciones (Bueno García, 2017). Esta formación se ha orientado a dar respuesta pedagógica al hecho de tener que situar al estudiante en el foco del proceso de enseñanza/aprendizaje. Algunos de estos retos, han sido, por ejemplo:

- La adaptación de nuevas metodologías docentes necesarias para el desarrollo de distintos tipos de competencias (generales, trasversales, específicas, etc.), con sus correspondientes resultados de aprendizaje y, por tanto, los procedimientos adecuados para su evaluación.

- La integración pertinente de los avances en tecnologías de la información y comunicación, como herramienta de aprendizaje de competencias y su relación con los procesos de innovación docente.

- La atención a la diversidad de estudiantes, que poseen diversos perfiles (itinerarios formativos previos, estrategias de procesamiento de la información, intereses, etc.). Cada vez es mayor el número de estudiantes procedentes de Programas ERASMUS, SICUE, y otros programas internacionales; además de estudiantes con necesidades especiales, dentro de una concepción de Universidad inclusiva.

- La gestión docente que hoy nos obliga a tener que abandonar el individualismo para trabajar en equipo y cooperativamente con el resto de equipo docente.

Del mismo modo, que parece que la evaluación docente puede incidir en la mejora de la docencia, a través de un apoyo a los programas de formación permanente del profesorado, también puede ser una herramienta para promover la necesaria innovación. Evaluación y formación pueden ser dos incentivos para promover procesos de innovación. Sin embargo, hemos de ser conscientes de la complejidad 
de la generación de los procesos de innovación, entendido como cambios que promueven mejoras y que deberían ser liderados desde contextos institucionales de departamento o titulación para que perdure su efecto (Rodríguez Espinar, 2013a, p. 123).

Para finalizar, queríamos recoger también las implicaciones o consecuencias que la evaluación de la actividad docente, a través del programa DOCENTIA, puede tener en el reconocimiento profesional. En este sentido, también el informe de expertos europeos antes mencionado (European Commission, 2013, p. 37), en su recomendación 6 indica: "Heads of institutions and institutional leaders should recognise and reward (e.g. through fellowships or awards) higher education teachers who make a significant contribution to improving the quality of teaching and learning, whether through their practice, or through their research into teaching and learning".

Ante este dilema, se plantea una reflexión: ¿Por qué debería un profesor dedicar una parte importante de su tiempo y esfuerzo personal y profesional a mejorar la experiencia de aprendizaje de sus estudiantes, cuando existe una brecha de reputación continua entre la investigación y la enseñanza y la falta de respaldo institucional son importantes desincentivos para el desarrollo de una cultura de calidad de la docencia? Entre los incentivos académicos en Universidad, aun no se conoce bonificación por la participación destacada de la docencia. Y a esto añadimos, por otro lado, que la mayoría de los rankings universitarios internacionales se encuentran sesgados hacia los índices de citas de publicaciones de investigación más fáciles de contabilizar, en lugar de mirar la misión universitaria más amplia en áreas tan fundamentales como la enseñanza y el aprendizaje, que son menos susceptibles de una medida objetiva.

La enseñanza de calidad, a diferencia de la investigación de calidad, no conduce a resultados fácilmente verificables. Mejorar el prestigio de una institución universitaria por la calidad de su docencia y proporcionar incentivos a sus profesores, requiere un esfuerzo adicional a las autoridades universitarias, que parece de momento, al menos complejo de conseguir. Sin embargo, la experiencia 
acumulada en programas como DOCENTIA nos ofrecen un camino por el que seguir avanzando en, al menos, un reconocimiento equilibrado entre docencia e investigación universitaria.

\section{Algunos retos del programa DOCENTIA}

El cambio operado en el sistema universitario español con la incorporación de instituciones con una oferta educativa exclusivamente online, la ya mencionada orientación de las enseñanzas hacia los estudiantes y la necesidad de alcanzar un mayor nivel de profesionalización de la actividad docente reclaman una reorientación del programa DOCENTIA, que permita hacer frente a los nuevos retos derivados de la evaluación de la actividad docente del profesorado universitario. Entre esos retos haremos mención aquí a dos que consideramos fundamentales: la evaluación de los equipos docentes y el desarrollo profesional del profesorado.

El programa DOCENTIA tendría que incorporar la posibilidad de evaluar una actividad docente desarrollada en equipo. La enseñanza es o se dirige hacia una labor desarrollada por equipos de personas. Especialmente en la enseñanza on line pero también en las modalidades presenciales y semipresenciales, la labor docente se desarrolla por un equipo compuesto por uno o varios profesores que planifican la enseñanza e imparten clases, acompañados por otros docentes que desarrollan labores de tutoría, por técnicos que diseñan y editan los materiales didácticos y acompañan a los profesores durante el desarrollo de las clases, por técnicos y/o profesionales que se ocupan de la supervisión de las actividades prácticas de la materia, etc.

El programa DOCENTIA tendría que afrontar la evaluación de la actividad docente desarrollada en el marco de una carrera profesional docente, por ahora desdibujada y carente de referencias en el sistema universitario español. La mejora de la actividad docente del profesorado universitario requiere un marco de referencia profesional que, mediante la evaluación, lo oriente desde su desempeño en un momento dado hasta la siguiente etapa de crecimiento. En este sentido, el programa DOCENTIA debería recoger un marco de referencia profesional y situarlo como 
criterio de evaluación del progreso en la carrera docente del profesorado. Elementos como la investigación sobre la propia práctica profesional deberían estar presentes en la nueva versión del programa. La propuesta elaborada por la Red Estatal de Docencia Universitaria (RED-U) (Paricio Royo, 2018) es un buen ejemplo del tipo de marco de referencia profesional que podrían utilizarse para evaluar y orientar el desarrollo docente del profesorado.

\section{Referencias bibliográficas}

ANECA (2015). Programa DOCENTIA. Programa de apoyo para la evaluación de la actividad docente del profesorado universitario. Integración y actualización de la documentación del programa 2015. v1 versión (recuperado de http://www.aneca.es/Programas-deevaluacion/DOCENTIA/Documentacion-del-programa).

ANECA (2017). Informe sobre el estado de la evaluación externa de la calidad en las universidades españolas. (recuperado de www.aneca.es/Programas-deevaluacion/DOCENTIA/Resultados).

Bueno García, C. (2017). Evaluación de la formación pedagógica del profesorado universitario. Universidad de Zaragoza, Zaragoza. Recuperado a partir de http://zaguan.unizar.es/record/65610

European Commission. (2013). Improving the quality of teaching and learning in Europe's higher education institutions. Bruxelas: Comision Europea. Recuperado a partir de http://ec.europa.eu/dgs/education_culture/repository/education/library/ reports/modernisation_en.pdf

Fernández March, A. (2008). La gestión de la formación del profesorado en la Universidad. Teoría de la educación, (20), 275-312.

González Such, J. (1998). Estudio de un instrumento para la evaluación del profesorado universitario (http://purl.org/dc/dcmitype/Text). Universitat de València. Recuperado a partir de https://dialnet.unirioja.es/servlet/tesis?codigo $=73416$

Lizasoain-Hernández, L., Etxeberria-Murgiondo, J., \& Lukas-Mujika, J. F. (2017). Propuesta de un nuevo cuestionario de evaluación de los profesores de la Universidad del País Vasco. Estudio psicométrico, dimensional y diferencia, 23(2), art. 1. https://doi.org/: httdoi.org/10.7203/relieve.2 3.2.10436

López Cámara, A. B. (2014). Diseño de un protocolo de evaluación de las competencias docentes del profesorado universitario (http://purl.org/dc/dcmitype/Text). Universidad de Córdoba. Recuperado a partir de https://dialnet.unirioja.es/servlet/tesis?codigo $=68177$ 
Marsh, H.W. (1987): Students' evaluations of university teaching: Research findings, methodological issues, and directions for future research. International Journal of Educational Research, 11, 253-388.

Miguel Díaz, F. M. de. (1999). El Plan Nacional de Evaluación de la Calidad de las Universidades: problemas y alternativas. Revista interuniversitaria de formación del profesorado, (34), 99-114.

Murillo Torrecilla, F. J. (2008). La Evaluación del Profesorado Universitario en España. Revista Iberoamericana de Evaluación Educativa, 1(3), 29-45.

Paricio Royo, J. (2018). Marco de desarrollo profesional del profesorado universitario. Planteamiento general y dimensiones. Zaragoza: REDU.

Peterson, K. (1984): Methodological problems in teacher evaluation. Journal of Research and Development in Education, 1 (4), 62-70.

Pozo Muñoz, C., Giménez Torres, M. L., \& Nieto, B. (2009). La Evaluación de la calidad docente en el nuevo marco del EEES: un estudio sobre la encuesta de opinión del Programa DOCENTIA-ANDALUCíA. XXI. Revista de educación, (11), 43-64.

PROGRAMA DOCENTIA. (2016). Autorinform Institucional de Meta-Evaluación. 8a convocatoria: curso 2015-2016. Universidad de Salamanca. Recuperado a partir de http://qualitas.usal.es/docs/INF2.2_2016_02_InfMetaevaluacion_15_16_ap robado_comision.pdf

Rodríguez Conde, M. J. (1994). El autoinforme en la evaluación docente del profesorado universitario (http://purl.org/dc/dcmitype/Text). Universidad de Salamanca. Recuperado a partir de https://dialnet.unirioja.es/servlet/tesis?codigo=130789

Rodríguez Conde, M. J. (2011). La garantía de la calidad, base de la movilidad. REDU: Revista de Docencia Universitaria, 9(3), 99-117.

Rodríguez Espinar, S. (2013a). La evaluación de la calidad en la educación superior: fundamentos y modelos. Madrid: Editorial Síntesis.

Rodriguez Espinar, S. (2013b). Panorama internacional de la evaluación de la calidad en la educación superior. Madrid: Editorial Síntesis. Recuperado a partir de https://www.sintesis.com/biblioteca\%20de\%20educaci\%C3\%B3n145/panorama\%20internacional\%20de $\% 201$ la $\% 20$ evaluaci\%C3\%B3n\%20 de\%20la\%20calidad\%20en\%20la\%20educaci\%C3\%B3n\%20superiorebook-1756.html

Stake, R., Contreras, G., \& Arbesú, I. (2011). Evaluando la calidad de la universidad, particulamente su docencia. Perfiles Educativos, 33(número especial), 155168.

Standards and Guidelines for Quality Assurance in the European Higher Education Area (ESG). (2015). Brussels, Belgium.

Tejedor Tejedor, F. J. (1990). La evaluación del profesorado en la Universidad de Santiago. Revista española de pedagogía, 48(186), 337-364.

Tejedor Tejedor, F. J., \& Jornet Meliá, J. M. (2008). La evaluación del profesorado universitario en España. REDIE: Revista Electrónica de Investigación Educativa, 10(1), 5. 
Vidal, J. (1999). Plan nacional de evaluación de la calidad de las universidades. Indicadores en la universidad: información y decisiones. Madrid: Secretaría General Técnica. Centro de Publicaciones. Ministerio de Educación y Cultura. Recuperado a partir de https://sede.educacion.gob.es/publiventa/ImageServlet?img=E-7873.jpg

Villa Sánchez, A. (1985). La evaluación del profesor: perspectivas y resultados. Revista de educación, (277), 55-93. 